Щ्)FRANÇAISE

$\gg \mathrm{DE}$

필 PEAGOGIE
Revue française de pédagogie

Recherches en éducation

154 | janvier-mars 2006

La construction des politiques d'éducation : de nouveaux rapports entre science et politique

\title{
Brougère Gilles \& Rayna Sylvie (dir.). Accueillir et éduquer la petite enfance. Les relations entre parents et professionnels
}

Lyon : INRP, 2005. - 209 p. (Éducation, politique, sociétés)

\section{Pascale Garnier}

\section{(2) OpenEdition}

Journals

Édition électronique

URL : http://journals.openedition.org/rfp/168

DOI : $10.4000 /$ rfp. 168

ISSN : 2105-2913

Éditeur

ENS Éditions

Édition imprimée

Date de publication : 1 mars 2006

Pagination : 199-200

ISBN : 2-7342-1039

ISSN : 0556-7807

Référence électronique

Pascale Garnier, «Brougère Gilles \& Rayna Sylvie (dir.). Accueillir et éduquer la petite enfance. Les relations entre parents et professionnels », Revue française de pédagogie [En ligne], 154 | janvier-mars 2006, mis en ligne le 14 octobre 2010, consulté le 21 septembre 2020. URL : http:// journals.openedition.org/rfp/168; DOI : https://doi.org/10.4000/rfp.168

Ce document a été généré automatiquement le 21 septembre 2020.

(c) tous droits réservés 


\title{
Brougère Gilles \& Rayna Sylvie (dir.). Accueillir et éduquer la petite enfance. Les relations entre parents et professionnels
}

\author{
Lyon : INRP, 2005. - 209 p. (Éducation, politique, sociétés) \\ Pascale Garnier
}

\section{RÉFÉRENCE}

Brougère Gilles \& Rayna Sylvie (dir.). Accueillir et éduquer la petite enfance. Les relations entre parents et professionnels. Lyon : INRP, 2005. - 209 p. (Éducation, politique, sociétés)

Co-dirigé par Sylvie Rayna et Gilles Brougère, l'ouvrage s'inscrit dans un domaine de recherche, la petite enfance, qu'ils ont largement contribué à développer par leurs propres travaux et collaborations. Issu d'un séminaire organisé à l'INRP, il réunit six études portant sur les conceptions et les pratiques d'accueil des jeunes enfants autour d'une thématique commune: les relations entre parents et professionnels. L'intérêt majeur de l'ouvrage est d'en montrer l'actualité et la forte diversité : tant au niveau des institutions étudiées en France (crèche collective, école maternelle et pouponnière sociale), que des cultures et des politiques publiques nationales. Du point de vue des parents, privilégié dans la plupart de ses contributions, cette diversité est également mise en valeur, que ce soit leurs attentes à l'égard des institutions, leurs investissements et modalités de communication avec les professionnels, leurs représentations de la petite enfance et celles du devenir de leur enfant. Qui dit diversité dit non seulement pluralité des dispositifs, des acteurs, de leurs stratégies et modalités d'engagement, mais aussi complexité de leurs relations. D'où l'importance de l'introduction, " Parents et professionnels face à l'accueil et à l'éducation du jeune enfant ", 
où $\mathrm{G}$. Brougère pose une série de questions qui traversent peu ou prou les six travaux présentés.

2 Impossible, en effet, de ne pas interroger la frontière qui constitue deux catégories bien distinctes d'acteurs : les uns désignés comme des "professionnels ", les autres comme des «parents ». Les avancées difficiles d'une professionnalisation de ces métiers dits «maternels » montrent tout l'effort fait pour construire des qualifications et des standards doublement abstraits de la singularité et d'une naturalisation des relations mère-enfant. Il faut également insister ici sur la «professionnalisation du rôle de la mère », soulignée dès 1973 par le travail pionnier de J.C. Chamboredon et J. Prévot, à laquelle notre présent associe désormais les pères. Aussi, chaque institution témoigne à sa manière de ce type de partage, frontière à chaque fois retracée par ses acteurs. Une telle analyse est indispensable pour comprendre ce qui se joue dans l'idée même de "partenariat» devenue aujourd'hui une injonction institutionnelle qui s'impose aux familles et aux professionnels. De part et d'autre, elle recouvre des pratiques et des significations très hétérogènes, des rationalités proprement politiques aussi bien que gestionnaires et éducatives. Son principe même qui suppose un espace de choix entre différents modes et lieux d'accueil est mis à l'épreuve par les situations auxquelles sont confrontées les familles. Pour les professionnels, cette thématique du partenariat connaît une nouvelle inflexion qui met l'accent sur la parentalité, l'accueil conjoint des parents et des enfants, voire sur une éducation familiale. Plus avant, cette présentation appelle à questionner l'objectif même d'une relation partenariale : pourquoi ? pour qui ? C'est dire l'hétérogénéité des démarches d'accueil des jeunes enfants que recouvrent des terminologies flottantes : soins (approche physiologique, hygiénique et pédiatrique), éveil, éducation, socialisation, scolarisation... Au cœur des relations entre parents et professionnels se jouent donc différentes conceptions de l'enfant et de son intérêt : santé, développement personnel et social, réussite scolaire...

3 L'ouvrage s'ouvre par «L'examen thématique de l'OCDE des politiques d'accueil et d'éducation de la petite enfance ", présenté par Sylvie Rayna et John Benett. Au niveau des treize pays de l'OCDE de cette enquête lancée en 1998, les auteurs montrent comment le renforcement de l'implication des familles constitue désormais un élément majeur des politiques destinées à favoriser un accès équitable des jeunes enfants à une éducation de qualité. Ils font également état de ses obstacles en termes de «barrière culturelle » et de disponibilité des familles.

4 Véronique Francis s'attache au "Parternariat école-famille: le rôle de l'enfant messager " pour étudier les rapports que les familles populaires entretiennent à l'école maternelle. Pour serrer au plus près les pratiques, elle examine des dispositifs d'information à caractère conversationnel qui circulent entre la classe et la famille, tels les « cahiers de vie » qui témoignent de l'activité de l'enfant à l'école. Ainsi, elle peut mettre en lumière et analyser tout le travail des mères pour avoir accès, de cette manière indirecte, aux contenus et aux démarches mis en œuvre dans la classe. C'est, du même coup, l'importance de l'enfant en tant que messager et des interactions verbales qui se nouent grâce à ces dispositifs qui est soulignée. Sa réflexion sur la diversité des pratiques enseignantes permet également de pointer les ambiguïtés qui traversent ces dispositifs. Cette étude insiste au total sur les moyens qu'offre ou non l'école maternelle aux familles populaires pour non seulement savoir décrypter ses messages explicites et implicites, mais aussi pour avoir prise sur elle ; c'est-à-dire en un mot, pour devenir véritablement des « parents d'élèves ». 
5 Dans «Suppléance précoce et coopération entre parents et professionnels: l'exemple des pouponnières sociales en France ", Marie-Pierrre Mackiewicz nous plonge au cœur des évolutions récentes en matière de protection de l'enfance dont la coopération avec les familles est désormais un enjeu majeur. Elle construit un modèle de la relation de suppléance, relatif aux configurations familiales des enfants accueillis. Cinq modes de suppléance sont ainsi distingués qui vont de pair avec des rapports différents aux professionnels et des implications différentes des parents dans les processus de décision. S'élargissant à la notion de relation de co-éducation, l'analyse interroge comment elle peut s'accompagner d'une dynamique de requalification des parents, quand les pouponnières sociales figurent encore un lieu de stigmatisation des familles.

6 Les deux travaux suivants, celui de Catherine Bouve, Relations parents professionnels dans les crèches collectives : une analyse du point de vue des parents, et celui de Pierre Moisset, La diversité des rapports parentaux à la crèche et ses déterminants, présentent deux modes de construction des relations entre parents et crèche collective. Le premier s'attache à construire une typologie des relations avec les professionnels combinant le type d'échanges et d'adhésion aux normes éducatives de la crèche. Il montre différents profils parentaux irréductibles à une catégorisation socioéconomique des familles signifiant distance ou proximité à l'institution. Construite à partir de la notion de "culture pédiatrique ", la typologie du second insiste en outre sur la diversité des personnels dans les crèches où les auxiliaires de puériculture et les psychologues constituent des interlocuteurs bien différenciés pour les parents.

7 L'arrivée des bébés, le matin, dans les crèches françaises et japonaises: une comparaison des pratiques d'accueil, étude réalisée par Olga Baudelot, Miwako Hoshi-Watanabe et Hiroyo Takahashi, développe un travail d'observation comparative des pratiques quotidiennes dans les crèches. L'analyse minutieuse des dispositifs, des lieux, des temporalités, des gestes et des modalités d'interactions, permet de mettre en évidence deux modalités d'accueil fortement contrastées: en France, centrée sur la relation parentprofessionnel et la question de la séparation pour l'enfant; au Japon, privilégiant la proximité physique avec l'enfant et la recherche d'un prolongement maternel avec la famille. Au-delà de la diversité des modes de prise en charge d'un pays à l'autre, se révèlent ainsi des traditions éducatives et des conceptions différentes des jeunes enfants.

8 Une large bibliographie nationale et internationale rassemble l'ensemble des références citées par les six études de cet ouvrage : outil précieux pour se repérer dans ce domaine des recherche sur la petite enfance, outil également fédérateur des différentes méthodologies et choix théoriques des auteurs. Malgré leur hétérogénéité, la perspective frayée par cet ouvrage s'inscrit nettement dans une approche sociologique soucieuse d'articuler le positionnement des acteurs, le sens des pratiques et leur mise en forme institutionnelle, politique et culturelle. Dans cette perspective, l'accent pourrait être également mis sur les dynamiques des rapports entre les parents et professionnels et sur leurs transformations à mesure que l'enfant grandit. En effet, si des profils des parents sont bien diversifiés et échappent à tout déterminisme social, il reste à comprendre comment ils sont mis à l'épreuve des situations auxquelles les familles sont confrontées, donnant lieu à des réajustements, voire à de nouvelles postures. L'ouvrage appelle ainsi une analyse de cette expérience que d'être "parent ", dans la durée, à travers la confrontation aux différents milieux où est accueilli leur 
enfant. Avec ces transformations, place peut être faite également à une représentation de l'enfant comme "acteur " de ces relations entre parents et professionnels et à son expérience vécue. Aussi, bien présente quand il s'agit d'observer les pratiques françaises et japonaises d'accueil des jeunes enfants dans les crèches, l'analyse comparative dont chacun connaît l'extrême difficulté, mériterait sans doute d'être davantage avancée sur le terrain des différentes institutions françaises investiguées. C'est dire, d'une autre manière, la richesse foisonnante de cet ouvrage, l'originalité des travaux et la qualité des matériaux qu'il réunit.

\section{AUTEURS}

\section{PASCALE GARNIER}

Institut universitaire de formation des maîtres, Créteil ESCOL, université Paris VIII 